\title{
Molecular Rotor based on an Oxidized Resorcinarene
}

\author{
Daniel T. Payne, ${ }^{1,2, *}$ Jan Labuta, ${ }^{1, *}$ Zdenĕk Futera, ${ }^{3}$ Václav Březina, ${ }^{4}$ Lenka Hanyková, ${ }^{4}$ \\ Mandeep K. Chahal, ${ }^{1}$ and Jonathan P. Hill ${ }^{1, *}$
}

\author{
${ }^{1}$ WPI Center for Materials Nanoarchitectonics, National Institute for Materials Science, Namiki 1-1, Tsukuba, \\ Ibaraki 305-0044, Japan. \\ ${ }^{2}$ International Center for Young Scientists, National Institute for Materials Science, Namiki 1-1, Tsukuba, \\ Ibaraki 305-0044, Japan. \\ ${ }^{3}$ Institute of Physics, Faculty of Science, University of South Bohemia, Branišovská 1760, České Budějovice \\ 370 05, Czech Republic \\ ${ }^{4}$ Faculty of Mathematics and Physics, Charles University, V Holešovičkách 2, 18000 Prague 8, Czech Republic \\ E-Mail: Payne.Daniel@nims.go.jp, Labuta.Jan@nims.go.jp and Jonathan.Hill@nims.go.jp
}




\section{Abstract}

Molecular single stator-double rotor activity of an oxidized resorcinarene (fuchsonarene) macrocycle containing unsaturated hemiquinonoid groups at its meso positions was investigated. Fuchsonarenes containing two hemiquinonoid substituents at diagonally-opposed meso-positions with two electron rich phenol groups at the remaining meso-positions between the hemiquinonoid groups. All meso-substituents are in proximity at one side of the resorcinarene macrocycle (so-called rccc-type isomer) with rotational activity of the phenol meso-substituents. Rotation rates of the phenol moieties can be controlled by varying temperature, solvent polarity and acidity of the medium of study with rotation being thermally activated in neutral and acidic media and tunable in the range from $2 \mathrm{~s}^{-1}$ to $20,000 \mathrm{~s}^{-1}$. Experimental and computational data indicate that rotation of the mobile phenol meso-substituents is remotely affected by interactions with acidic solvents at the carbonyl $\mathrm{C}=\mathrm{O}$ groups of macrocyclic acetyloxy groups, which occurs with the emergence of a lower energy electronic absorption band whose intensity is correlated with both the acidity of the medium and the rotation rate of the phenol substituents. Time-dependent DFT calculations suggest that the low energy band is due to a molecular conformational adjustment affecting electronic conjugation caused by strong interaction of macrocyclic acetyloxy carbonyl groups with the acid medium. The work presents a molecular mechanical model for estimating solution acidity and also gives insight into a possible method for modulating rotor activity in molecular machines. 


\section{TOC Graphic}
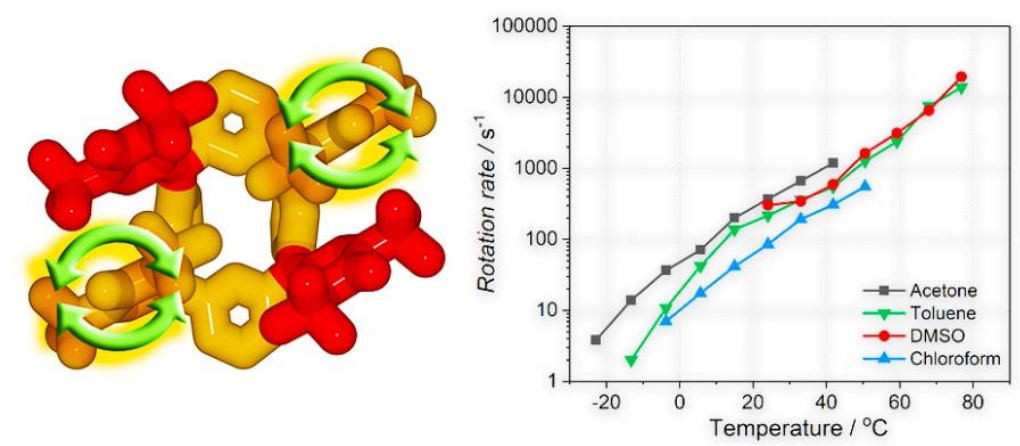

\section{Introduction}

Molecular rotors ${ }^{1-7}$ are a class of molecular machine ${ }^{8-13}$ containing two key components, the stator and the rotor, which undergo facile relative rotation. ${ }^{14}$ The operation of rotors might be an important factor in the development of synthetic molecular machines where they would be integrated with other molecular components having different operational activities. ${ }^{16,17}$ Also, there exist several examples of biological molecular machine where a rotor molecular element operates based on chemical and physical stimuli. ${ }^{18-20}$ These can be treated as archetypes for the development of molecular- and nanomolecular-level machine architectures. Although substantial progress has been made in the area of molecular machine synthesis, ${ }^{21,22}$ and there have been demonstrations of important features of rotors such as unidirectional motion ${ }^{23-25}$ or supramolecular activity, ${ }^{26}$ there remains the requirement to investigate different molecular geometries and molecule-level activity in order to establish design rules for each active element of any possible molecular machine assemblies. A useful medium in this respect has been the metal-organic-frameworks (MOFs), where 
the rotation especially of the organic linker components presents the possibility to study substituent rotation under low density conditions. ${ }^{27,28,29}$ Apart from the molecular machine aspects of rotors, there already exist several applications of molecular rotors as probes of local conditions in biological systems, or as sensors. ${ }^{30-32}$ Rotor activity signified by variation in fluorescence output wavelength of certain molecule types can be harnessed to reveal local viscosity in biological membranes or models thereof, ${ }^{33-36}$ while rotation rate modulation also signalled by fluorescence emission characteristics can also be used to indicate the presence of analytes. ${ }^{37-39}$ Molecules having several proximal component moieties are suitable archetypes of rotors because of intramolecular interactions that govern the mutual motion of substituents. However, although macrocycles such as porphyrins ${ }^{40-42}$ and calixarenes $^{43}$ or highly-substituted hydrocarbons ${ }^{44,45}$ have been extensively used, resorcinarenes ${ }^{46-50}$ have hardly been studied for this purpose, and in cases where they have been used, they are usually operating only as a supramolecular host where an encapsulated guest rotates. ${ }^{51,52}$

Resorcinarenes (Figure 1) are an intensively studied class of macrocycles, which can be readily synthesized in high yields by the simple condensation of resorcinol with an aldehyde. ${ }^{53}$ They are sterically crowded molecules and can exist in many different isomeric forms due to the relative orientations of their hindered substituents. ${ }^{54}$ Of the common isomers denoted $r c t t, r c c c, r c c t, r t c t$, the rccc forms (also called 'cone'; see Supplementary Information for structures) have been studied due to their propensity to form oligo-molecular capsules with host-guest ${ }^{46-50,55,56}$ and catalytic properties. ${ }^{57-59}$ However, the effect of meso-substituent identity on the chemical properties of resorcinarene macrocycles has not been investigated in any depth despite the ease of synthesis of the relevant compounds and the possibility of assessing inter- and intramolecular processes based 
on the isomeric structures of the compounds. This has been due to the widespread interest in the chemistry of the carcerand structures and the essential chemistry of the resorcinarene macrocycle itself has been largely neglected. ${ }^{60,61}$ We have recently reported a class of resorcinarenes containing unsaturated meso-positions for which we have applied the term 'Fuchsonarenes' (Figure 1) since these macrocycles contain components similar to fuchsone dyes. ${ }^{62}$ The compounds have so far been studied for their activity as multimodal molecular switches ${ }^{63}$ or as photosensitizers for singlet oxygen generation. ${ }^{64}$ Synthesis of the fuchsonarenes is enabled by the introduction of several oxidizable groups at their meso-positions, i.e., anti-oxidant 3,5-di-tert-butyl-4-hydroxyphenyl groups (DtBHP), which allows hybridization at the meso-positions to be transformed to $s p^{2}$ following oxidation, introducing electronic conjugation between macrocycle and the resulting hemiquinonoid meso-substituents, and substantially rigidifying their structures. We had previously applied this principle to the meso-tetraphenylporphine system..$^{65-73}$ The nanometric dimensions, rigid conformations and chemical stability of the resulting fuchsonarene molecules suggest their potential as supramolecular components of functional nanostructures. This potential is enhanced by the already demonstrated possibility of synthesising different isomer forms (e.g., rctt and rccc) or by functionalisation at the macrocyclic resorcinol moieties. 

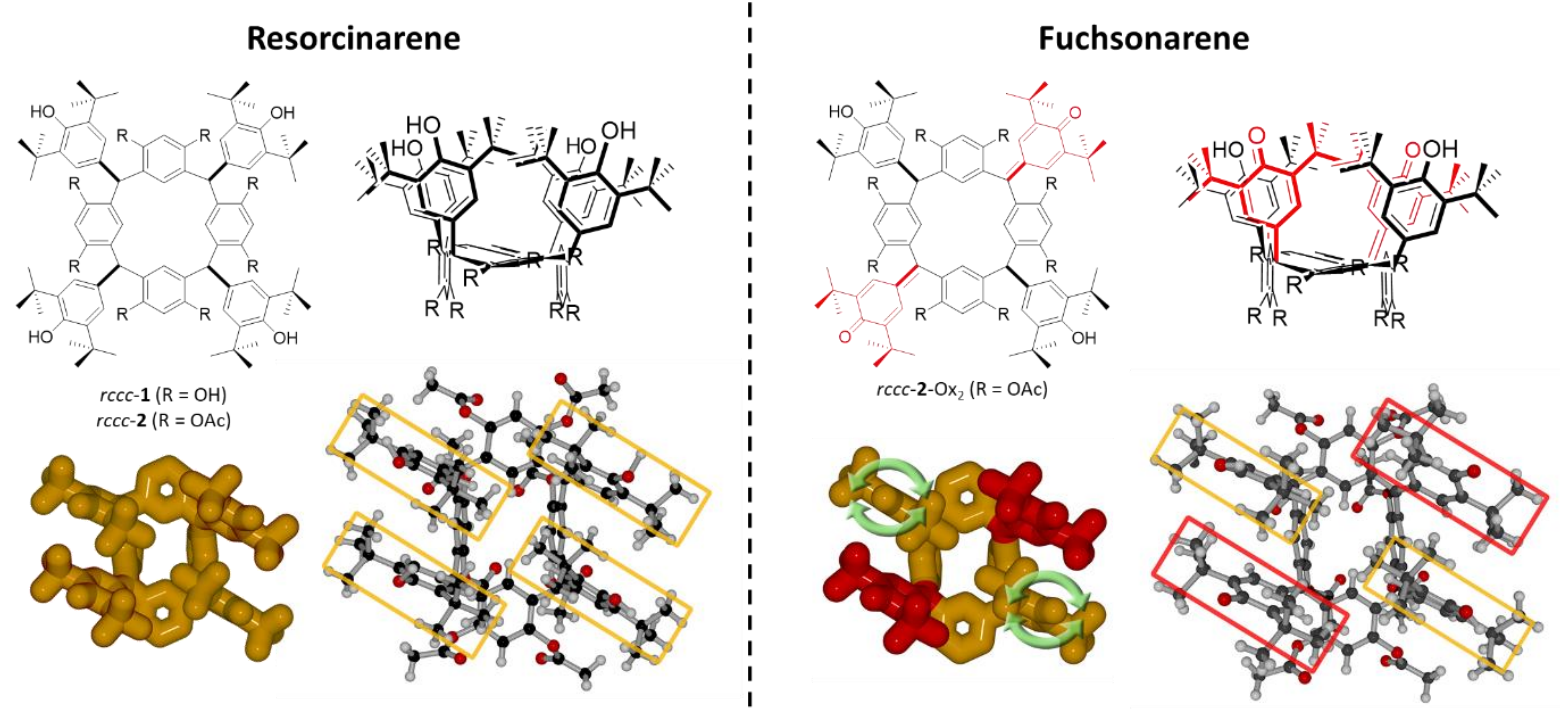

Figure 1. Chemical structures of resorcinarenes (left) and the corresponding fuchsonarenes (right) used in this work. Top: 2D and 3D line structures of the compounds. Bottom: 3D depictions of rccc-1 and rccc-2-Ox 2 derived from X-ray crystallographic data. ${ }^{63,64}$ Green arrows denote rotational activity of the phenolic meso-substituents in rccc-2-Ox $_{2}$. Antioxidant DtBHP substituents are indicated in yellow frames, while hemiquinonoid groups are denoted in red frames.

Here we describe a system that exploits not only the rigidification of the resorcinarene macrocycle but also the rccc conformer in the formation of a fuchsonarene-based doublerotor/stator where the specific configuration of its substituents allows observation and study of the rotational motility of those substituents. Rotation rates of the rotors relative to the stator can be controlled between $2 \mathrm{~s}^{-1}$ and $>20,000 \mathrm{~s}^{-1}$ by varying the solvent, temperature or acidity. These effects were studied by ${ }^{1} \mathrm{H}$ NMR spectroscopy and electronic absorption (UV-vis) spectrophotometry. The latter was used to demonstrate that rotation rate can be probed optically where acidity of the solvent was applied as the controlling factor. Rotation rates and mechanisms were also investigated computationally using density functional theory (DFT), revealing good agreement between experimental and computed scenarios. 


\section{Results}

The resorcinarene synthesis involving condensation between resorcinol and a benzaldehyde, the rccc isomer of $\mathbf{1}$ is the thermodynamically favoured product and is available in increasing proportion over the kinetically favoured product ( $r c t t$ isomer) at depressed reaction temperatures. ${ }^{53}$ For 1, a 2.6:1 mixture of rctt:rccc was obtained when carrying out the synthesis at room temperature. Isomers were separated by per-O-acetylation (selectively at resorcinol-type hydroxyl groups) followed by fractional crystallization; rctt-2 precipitates rapidly from the acetylation reaction mixture while rccc- $\mathbf{2}$ crystallizes more slowly over a period of several days with further subsequent purification performed by using column chromatography. A full description of the synthetic methods can be found in the Supplementary Information. Oxidation of rccc-2 follows the same trend as previous studies, ${ }^{63,64}$ where double oxidation of rccc isomers is preferred, yielding the fuchsonarene derivative $r c c c-2-O x_{2}$, whose properties as a double-rotor/stator are described here.

Significant line broadening of the resonances due to certain meso substituent $t$-butyl groups in ${ }^{1} \mathrm{H}$ NMR spectra obtained at room temperature in chloroform-d $\left(\mathrm{CDCl}_{3}\right)$ was observed (Figure $2 a, b)$. This is assigned as being due to restricted rotation of the remaining non-oxidized phenolic meso-substituents of $r c c c-2-O x_{2}$. This had not previously been observed in ${ }^{1} \mathrm{H}$ NMR spectra of other fuchsonarene molecules and is so far a unique observation for this isomer structure and oxidation state, prompting our further investigation. An assignment of the NMR spectra of $r c c c-2-O x_{2}$ in $\mathrm{CDCl}_{3}$ (with TMS reference at room temperature) was achieved by a combination of 2D NMR spectra (at $50{ }^{\circ} \mathrm{C}$ ) with ${ }^{1} \mathrm{H}$ NMR spectra measured at various temperatures $\left(\mathrm{VT}-{ }^{1} \mathrm{H}\right.$ NMR) (see Supplementary Information). 


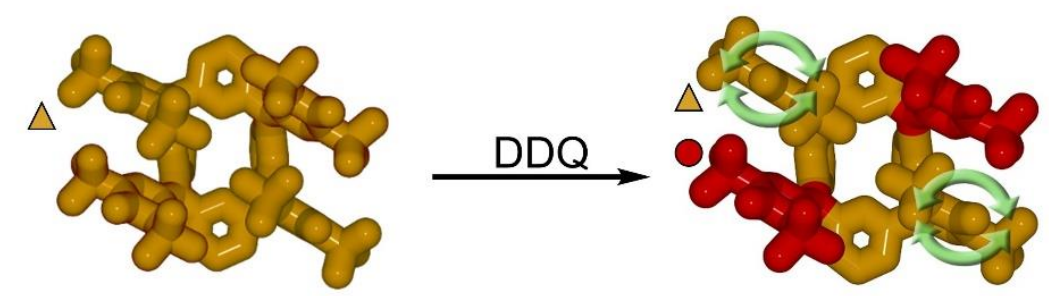

(a)

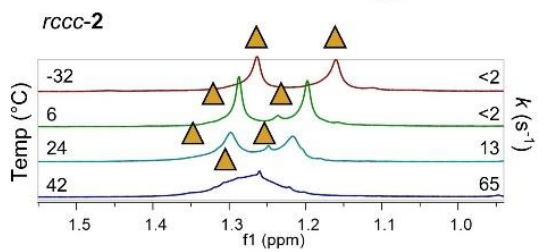

(c)

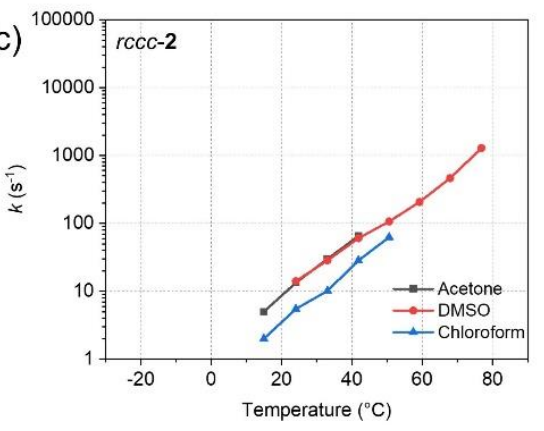

(e)
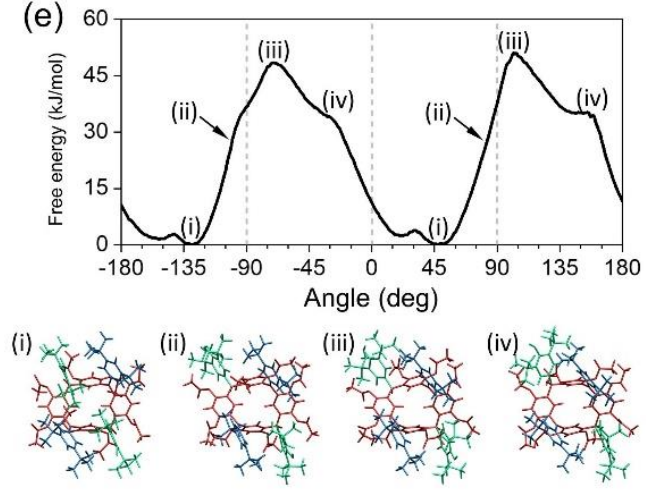

(b)

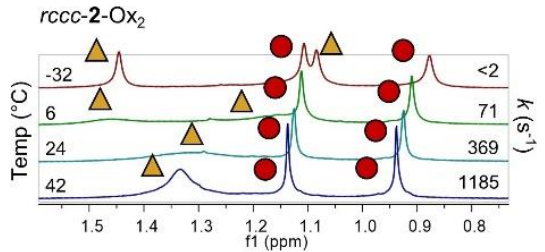

(d)

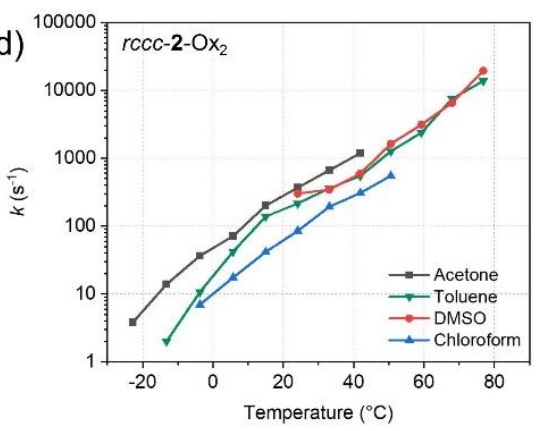

(f)

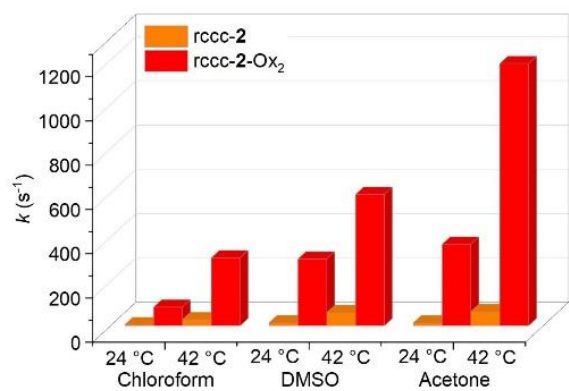

Figure 2. Relative rotation of the meso-substituents of $r c c c-2-\mathrm{Ox}_{2}$. The model at the top depicts the oxidation of mesosubstituents where hemiquinonoid groups are shown in red in the model at right. Regions of the molecule unchanged by the oxidation process are shown in yellow in the scheme at the top (DDQ: 2,3-dichloro-5,6-dicyano-1,4-benzoquinone). (a,b) High field (t-butyl) region of ${ }^{1} \mathrm{H}$ NMR spectra in acetone- $d_{6}$ at different temperatures of (a) rccc-2 and (b) rccc-2-Ox . (c,d) Log-linear plot of rotation rates in different solvents at accessible temperatures of phenolic meso substituents in (c) rccc-2 and (d) rccc-2-Ox 2 . (e) Free energy profile of rotation of one of the phenolic meso substituent (the top-left one in shown structures) of $r c c c-2-0 x_{2}$ as obtained from MD simulations at $25{ }^{\circ} \mathrm{C}$. Computational snapshots of several of the 
structures in acetone are included. (f) Comparison of rotation rates of phenolic meso substituents in $r c c c-2$ and $r c c c-2-0 x_{2}$ in different solvents at $24^{\circ} \mathrm{C}$ and $42{ }^{\circ} \mathrm{C}$.

Variable temperature ${ }^{1} \mathrm{H}$ NMR $\left({ }^{1} \mathrm{H}\right.$ VT-NMR) spectroscopy, in conjunction with spectral lineshape analysis, allows for a detailed characterization of the rotation rate $(k)$ of phenolic meso substituents in the compounds studied here. The broadening and coalescence of spectral lines (e.g. Figure $2 a, b)$ can be described by invoking a two-state symmetrical exchange between $A$ and $B$ states of phenolic $t$-butyl groups close to and remote from the center of the macrocycle, respectively (Figure 2, top). The spectrum can then be derived from the exchange between two non-interacting spin singlets and fitted using the spectral line shape formula $S(\omega)$ expressed in Equation 1. ${ }^{71,74,75}$

$$
\begin{aligned}
& S(\omega)=M_{0} \frac{\alpha_{\mathrm{A}}+\alpha_{\mathrm{B}}+2 k}{\alpha_{\mathrm{A}} \alpha_{\mathrm{B}}-k^{2}} \\
& \alpha_{\mathrm{A}}=R_{2 \mathrm{~A}}+k-i\left(\omega-\omega_{\mathrm{A}}\right) \\
& \alpha_{\mathrm{B}}=R_{2 \mathrm{~B}}+k-i\left(\omega-\omega_{\mathrm{B}}\right)
\end{aligned}
$$

where $M_{0}$ is equilibrium magnetization, $R_{2 \mathrm{~A}}$ and $R_{2 \mathrm{~B}}\left(\right.$ in s$^{-1}$ ) are spin-spin relaxation rates of each state, $k\left(\right.$ in $\left.\mathrm{s}^{-1}\right)$ is the rate constant of exchange (i.e. $180^{\circ}$ rotation of phenolic meso substituent), $i$ is the unit imaginary number, $\omega_{\mathrm{A}}$ and $\omega_{\mathrm{B}}\left(\right.$ in $\mathrm{rad} \mathrm{s}^{-1}$ ) are resonance frequencies of each state when no exchange occurs $\left(k=0 \mathrm{~s}^{-1}\right)$, and $\omega\left(\right.$ in rad s $\left.\mathrm{s}^{-1}\right)$ is an independent variable connected to chemical shift $\delta$ (in ppm) by formula $\omega=2 \pi v_{0} \delta$, where $v_{0}$ (in $\mathrm{MHz}$ ) is the operating frequency of the spectrometer (in the present case $v_{0}=300.4 \mathrm{MHz}$ ). The real part of Equation 1 (i.e., the absorption spectrum ${ }^{71}$ ) is used for actual spectral fitting (see section ${ }^{1} \mathrm{H}$ NMR spectra fitting in Supplementary information).

There is a connection between the rate constant $k$ and the Gibbs energy of activation $\Delta G^{\ddagger}$ (i.e., the barrier height) in the form of the Eyring equation (Equation 2). ${ }^{76}$ 


$$
k=\frac{k_{\mathrm{Boltz}} T}{h} e^{-\frac{\Delta G^{\ddagger}}{R T}}=\frac{k_{\mathrm{Boltz}} T}{h} e^{-\frac{\Delta H^{\ddagger}}{R T}} e^{\frac{\Delta S^{\ddagger}}{R}}
$$

where $k_{\text {Boltz }}$ is the Boltzmann constant, $h$ is the Planck constant, $R$ is the gas constant and $T$ (in $\mathrm{K}$ ) is the absolute temperature. The Gibbs activation energy $\Delta G^{\ddagger}=\Delta H^{\ddagger}-T \Delta S^{\ddagger}$, where $\Delta H^{\ddagger}$ is the enthalpy of activation and $\Delta S^{\ddagger}$ is the entropy of activation of the $180^{\circ}$ rotation process of the phenolic meso substituent. $\Delta H^{\ddagger}$ and $\Delta S^{\ddagger}$ were obtained using the analyses of temperature dependency of the rate constant $k$ using an Eyring plot (i.e., a plot of $\ln (k / T)$ vs. $1 / T$ with subsequent extraction of slope and intercept of the linear dependency). The results $\left(k, \Delta H^{\ddagger}\right.$ and $\left.\Delta S^{\ddagger}\right)$ are summarized in Tables S1, S2 for rccc-2 and Tables S3, S4 for $r c c c-2-\mathrm{Ox}_{2}$ with groups of ${ }^{1} \mathrm{H}$ NMR resonances used to obtain the data defined in Figures S1,S2 (marked by green triangles).

${ }^{1} \mathrm{H}$ VT-NMR studies on rccc-2 (Figure 2a, Table S1 and S2, Figures S3-S6) revealed that $180^{\circ}$ rotation of the meso substituents is slow $\left(13 \mathrm{~s}^{-1}\right)$ at room temperature in acetone- $d_{6}$ and that the barrier to rotation is high $\left(\Delta G^{\ddagger}=66.5 \mathrm{~kJ} \mathrm{~mol}^{-1}\right)$ at $24.1{ }^{\circ} \mathrm{C}$. The rate increases with temperature exponentially (i.e. straight lines in log-linear plot), and changing the solvent leads to variation in the rotation rates within a half order of magnitude, as shown in Figure 2c. For rccc-2-Ox 2 under identical conditions (Figure 2b, Table S3 and S4, Figures S7-S11), a similar pattern of rotation rate dependencies was found (Figure $2 \mathrm{~d}$ ). However, the rotation rate of the remaining unoxidized phenol substituents in $\mathrm{rccc}^{-2-O x_{2}}$ is significantly (more than one order of magnitude) greater than that of phenols in rccc-2 with, for example, a 28 -fold rate increase observed at $24{ }^{\circ} \mathrm{C}$ in acetone- $d_{6}$ (compare Figures $2 \mathrm{c}$ and $2 \mathrm{~d}$ or see Figure $2 \mathrm{f}$ ). This behaviour is counterintuitive considering the highly sterically hindered environments of the substituents since the unoxidized phenol groups are apparently blocked by the hemiquinonoid groups whose rotation is prevented by the double-bound connection 
of these substituents to the macrocyclic meso-positions. The barrier $\Delta G^{\ddagger}$ to the rotation of the phenolic meso substituents of $r c c c-2-O x_{2}$ was determined from NMR data to be $58.3 \mathrm{~kJ} \mathrm{~mol}^{-1}$ (in acetone- $d_{6}$, at $24{ }^{\circ} \mathrm{C}$, Table S4), which is in good agreement with the value obtained from its free energy profile (ca. $50 \mathrm{~kJ} \mathrm{~mol}^{-1}$ at $25^{\circ} \mathrm{C}$, Figure 2e) obtained using molecular dynamics (MD) simulations (see Computational Methods in the Supplementary Information and Figure S14). ${ }^{77-86}$ These values are similar to those found for 2-substituted biphenyls and meso-tetraarylporphyrins systems. ${ }^{87,88}$ These results demonstrate that oxidation of two meso-substituents of resorcinarene rccc-2 to fuchsonarene $r c c c-2-\mathrm{Ox}_{2}$ has the effect of promoting the rotation of the unoxidized phenolic meso substituents over those in rccc-2 and that the rate of rotation can be controlled by varying both the solvent used and the temperature, with rotation rates ranging from $<2 \mathrm{~s}^{-1}$ to $>20,000 \mathrm{~s}^{-1}$ depending on conditions (see Figure $2 \mathrm{c}, \mathrm{d}, \mathrm{f}$, Figure $\mathrm{S} 15$ ). The promoted rotation rate caused by oxidation at the meso-substituents is an interesting feature of this system which can be assigned to variation in the conformation of the macrocycle caused by $\mathrm{sp}^{2}$ hybridization at its meso positions. It is also an interesting possible design parameter when considering the preparation of rotor systems in similarly substituted molecules.

While variations in temperature and solvent can be used to control the rotation rate of meso-substituents in $r c c c-2-\mathrm{Ox}_{2}$, it was also found that exposure to trifluoroacetic acid-d (TFA- $d$ ) leads to a decrease in the rate of rotation of the unoxidized phenol meso substituents $\left(370 \mathrm{~s}^{-1}\right.$ in acetone- $d_{6}$ vs. $25 \mathrm{~s}^{-1}$ in TFA- $d$, Figure 3a) denoted by the appearance of two individual $t$-butyl resonances in the ${ }^{1} \mathrm{H}$ NMR spectrum. Interestingly, solutions of $r c c c-2-\mathrm{Ox}_{2}$ are orange in TFA, and the colour change is accompanied by a new absorption band centred at $517 \mathrm{~nm}$ in its UV-vis spectrum (Figure 3b). ${ }^{1} \mathrm{H}$ VT-NMR studies revealed that the rate of rotation in the presence of TFA 
can again be controlled by varying temperature, where rotation is essentially arrested below $6{ }^{\circ} \mathrm{C}$ but increases to $460 \mathrm{~s}^{-1}$ at $59^{\circ} \mathrm{C}$ (Figure 3c, Table S3, Figure S10, S15). This suggests that a third input for controlling the phenolic meso substituents rotation rate can be used, where exposure of the compounds to an acidic environment leads to a decrease in the rate of rotation.

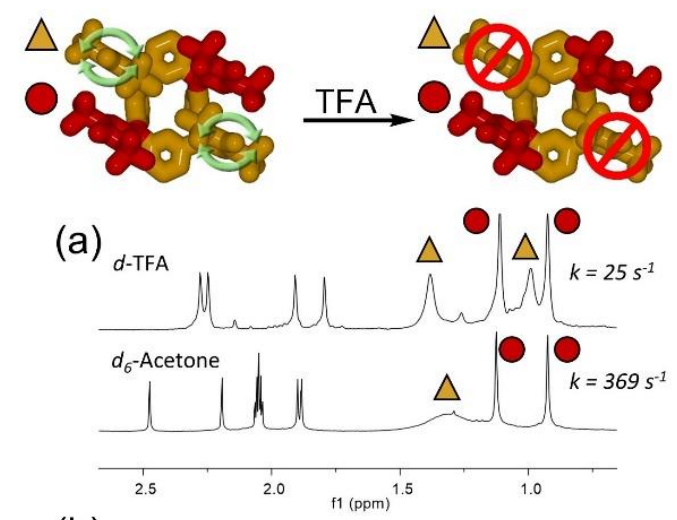

(b)
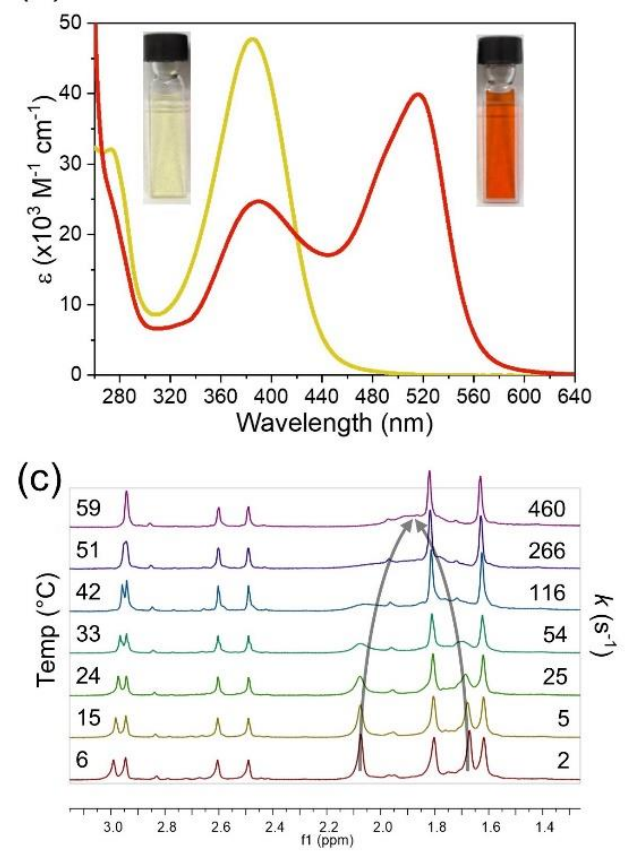

Figure 3. Effect of trifluoroacetic acid on the rate of rotation of phenolic meso substituents in $\operatorname{rccc}^{2}-\mathrm{Ox}_{2}$. (a) ${ }^{1} \mathrm{H}$ NMR spectra of $r c c c-2-\mathrm{Ox}_{2}$ in acetone- $d_{6}$ and TFA- $d$ at $24^{\circ} \mathrm{C}$ with symbols (triangle and circle) denoting different types of meso substituents. (b) UV-vis spectra of $r c c c-2-\mathrm{Ox}_{2}$ in chloroform (yellow) and TFA (red) at $3.05 \times 10^{-4} \mathrm{M}$ in a $1 \mathrm{~cm}$ pathlength 
cell. (c) ${ }^{1} \mathrm{H}$ VT-NMR spectra of $r c c c-2-\mathrm{Ox}_{2}$ in TFA- $d$ with spectra referenced to chemical shift of the first acetate resonance (2.48 ppm). Arrows indicate the coalescing resonances due to phenolic meso substituent.

The rotation rate of the unoxidized meso-substituents can be tuned under isothermal conditions by varying the composition of a binary mixture of TFA-d/acetone- $d_{6}$ (Figure 4, Table S5, Figures $\mathrm{S} 12, \mathrm{~S} 13)$. Increasing the ratio of TFA- $d$ in the binary mixture slows the rotation rate from ca. $470 \mathrm{~s}^{-1}$ to $10 \mathrm{~s}^{-1}$ (Figure 4a, Table S5) with a concomitant gradual change in the solution colour (Figure 4b) and variation in the UV-vis spectrum (Figure 4c). It should be noted that the barrier to the rotation $\Delta G^{\ddagger}$ of the meso-substituent increases in pure TFA to $65.6 \mathrm{~kJ} \mathrm{~mol}^{-1}$ (determined from NMR at $24{ }^{\circ} \mathrm{C}$, Table S4), which is again in reasonable agreement with the value of ca. $61 \mathrm{~kJ} \mathrm{~mol}^{-1}$ (at $25^{\circ} \mathrm{C}$, Figure S14) obtained from the free energy profile using MD simulations.

The presence of two isosbestic points at 401 and $427 \mathrm{~nm}$ (Figure 4c) in UV-vis spectra of solutions of varying TFA- $d$ /acetone- $d_{6}$ compositions and the results of singular value decomposition analysis (SVD) ${ }^{89-95}$ (Figure 4d, for details see Section 5 in Supplementary Information) indicate the presence of a third intermediate species. The spectra of second and third species qualitatively resemble those obtained by time-dependent density functional theory (TD-DFT) calculations (Figure 5b), which indicates some degree of interaction of the acid medium with $\mathrm{rccc}-2-\mathrm{Ox}_{2}$ (for more details, see Discussion). The predictable variation in UV-vis profile dependent on solvent composition (in this case, acid content) presents a molecular system whose rotation rate can be monitored photophysically using UV-vis spectrophotometry, allowing for the construction of calibration curves if ${ }^{1} \mathrm{H}$ NMR data can be correlated with the UV-vis data (Figure 4e). From this correlation, a set of calibration formulae can be generated that and used to indirectly determine the rate of rotation of 
the phenolic meso substituents based on the absorbance data (Figure 4e; for details see Section 6 in Supplementary Information).

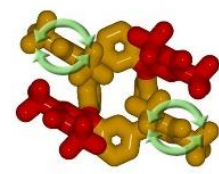

$0-100 \% d-$ TFA in $d_{6}$-Acetone

(a)

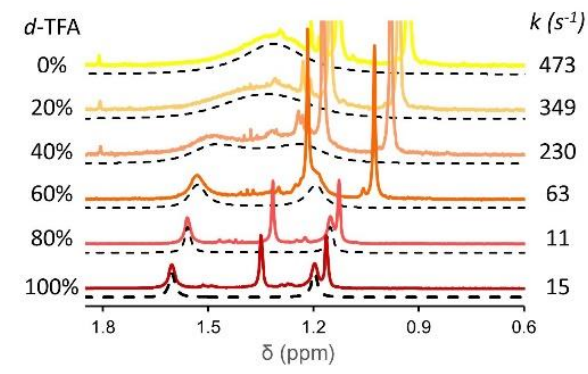

(b)

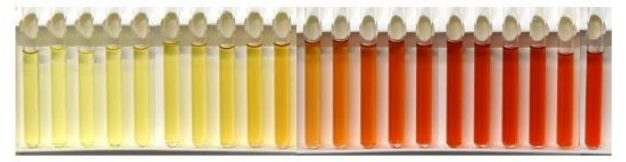

(c)

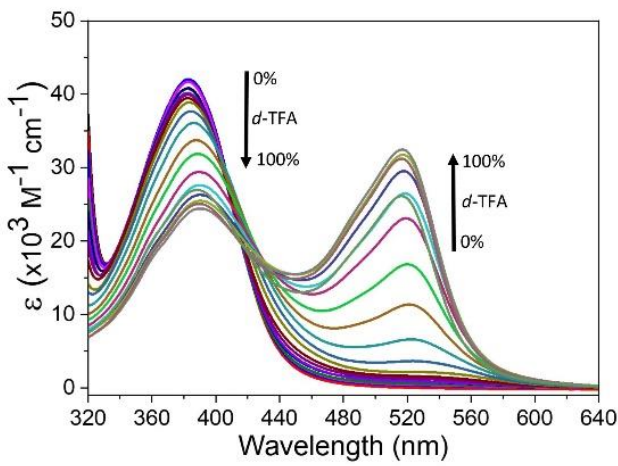

(d)
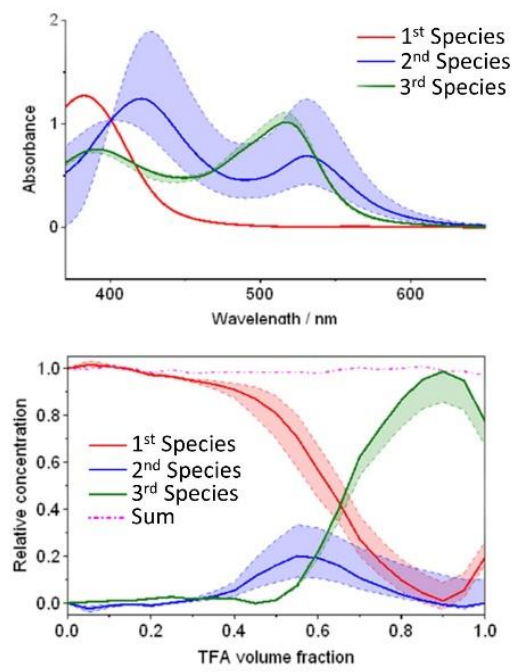

(e)

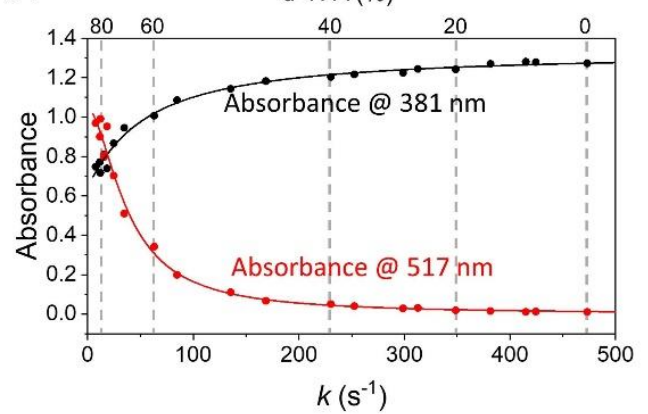

Absorbance@381nm Absorbance @ 517 nm

$$
\begin{gathered}
A_{381}=\frac{\alpha_{381}}{k^{\beta_{381}}+\gamma_{381}}+\delta_{381} \\
\alpha_{381}=-53.3 \\
\beta_{381}=1.09 \\
\gamma_{381}=78.1 \\
\delta_{381}=1.332
\end{gathered}
$$

Figure 4. Effect of TFA- $d$ volume fraction in acetone- $d_{6}$ on the rate of rotation of phenolic meso substituents in $r c c c-2$ -

$\mathrm{Ox}_{2}$. (a) ${ }^{1} \mathrm{H}$ NMR spectra of $r c c c-2-\mathrm{Ox}_{2}$ at $3.05 \times 10^{-4} \mathrm{M}$ in TFA- $d$ /acetone- $d_{6}$ mixtures at $24^{\circ} \mathrm{C}$. (b) Photographs of the samples prepared for this study indicating a gradual colour change. (c) UV-vis spectra of $r c c c-2-O x_{2}$ in TFA- $d /$ acetone- $d_{6}$ mixtures at $3.05 \times 10^{-4} \mathrm{M}$ in a $0.1 \mathrm{~cm}$ pathlength cell. (d) Summary of the SVD analysis indicating the presence of 3 
different species (for details, see Supplementary Information, Section 5). (e) Calibration curves and formulae constructed by correlating UV-vis and ${ }^{1} \mathrm{H}$ NMR data (for details, see Supplementary Information, Section 6).

\section{Discussion}

There are several interesting aspects of the $\operatorname{rccc}-\mathbf{2}-\mathrm{Ox}_{2}$ system presented here that have connotations for the properties of other similar molecular constructs. For example, many compounds containing multiple, especially conjugated, hemiquinonoid groups can also exist with an open shell electronic structure ${ }^{96,97}$ with unpaired electron density present at unsaturated oxygen atoms. In this case, we have excluded this possibility based on the only weak ESR response of rccc2-Ox (due to small amounts of phenoxyl radical always present in samples of these compounds, see Figure S16) and the thermally induced coalescence of the relevant ${ }^{1} \mathrm{H}$ NMR resonances, which must be a symptom of moiety rotation. Other work ${ }^{63,64}$ has shown that carbonyl $C=0$ bond lengths are consistent with closed-shell forms. Also, the changes in the hue of solutions where the acetone/TFA ratio is varied is reminiscent of solvatochromism. However, the variation is merely due to increases in absorbance of the new band at $520 \mathrm{~nm}$, and no shifts in absorption wavelength are observed, implying the emergence of another chemical entity with a different electronic structure.

Of the solvents employed in the present study, trifluoroacetic acid causes the most notable effects based on the emergent UV-vis band and rotation rates determined by NMR spectroscopy. Therefore, we must consider the effects of acid and possible protonation scenarios most closely. Protonation of fuchsone dyes such as 2,6-di-tert-butyl-4-(diphenylmethylene)cyclohexadien-1one $^{98}$ initially occurs at the carbonyl oxygen atom with subsequent rearrangement to a resonance stabilized methanide carbocation that has a characteristic ${ }^{13} \mathrm{C}$ NMR resonance at $\sim 200 \mathrm{ppm}$. 
However, for $r c c c-2-O x_{2}$ in TFA-d, no such low field carbocation resonance appears and the chemical shift of its hemiquinonoid carbonyl carbon atom is similar to that in $\mathrm{CDCl}_{3}$ solution (see Supplementary Information, Figures S17 \& S30 peak at low field: 186.5 ppm in both cases) indicating that protonation does not occur at hemiquinonoid groups of $r c c c-2-\mathrm{Ox}_{2}$ (for assignments of the NMR spectra of $r c c c-2-\mathrm{Ox}_{2}$ in different media see Supporting Information). Resistance to protonation by rccc-2-Ox 2 is most likely due to the low electron donating power of the resorcinarene acetyloxy ester groups so that rearrangement from protonated hemiquinone to meso-carbocation (which certainly occurs in the case of simple fuchsone ${ }^{98}$ ) is not supported, and the corresponding ${ }^{13} \mathrm{C}$ NMR resonance at low field is not found. ${ }^{13} \mathrm{C}$ NMR spectra of $r c c c-2-O x_{2}$ in TFA- $d$ do, however, reveal significant shifts in carbonyl resonances due to acetyloxy carbonyl groups (see Figure S17 \& S30; group of 4 peaks at 167-169 ppm $\left(\mathrm{CDCl}_{3}\right)$ shifts to $173-175$ ppm (TFA-d); see Fig. S29 for the corresponding ${ }^{1} \mathrm{H}$ NMR spectra). There are accompanying ${ }^{13} \mathrm{C}$ NMR shifts for the $\mathrm{sp}^{2}$-type meso-positions and the neighbouring cyclohexadienylidene substituent proton and carbon atoms (see Section 8, Supplementary Information) indicating that some structural change occurs close to the oxidized meso-positions, which is associated with acetyloxy/TFA interactions. These are the most important variations in the NMR spectra occurring in passing from non-polar $\left(\mathrm{CDCl}_{3}\right)$ to acidic (TFA-d $)$ solvent. This approximately $6 \mathrm{ppm}$ downfield shift of the acetyloxy $\mathrm{C}=\mathrm{O}{ }^{13} \mathrm{C}$ resonance (and associated NMR variations of oxidized meso-substituents) suggests some interaction with the acidic medium. Therefore, we have also investigated this using time-dependent density functional theory (TD-DFT) methods.

In TD-DFT, notably, while simple H-bonding interactions were observed not to generate low energy electronic absorption in $\mathrm{rccc}^{-2}-\mathrm{Ox}_{2}$ (Figure 5a), the constrained protonation of one of its 
acetyloxy $\mathrm{C}=\mathrm{O}$ group(s) was found to lead to the appearance of new lower energy bands in its TDDFT calculated UV-vis spectrum similar to those observed experimentally for solutions of $r c c c-2-O x_{2}$ in TFA (compare Figure 5b and Figure 3b). ${ }^{99-102}$ UV-vis spectra of $r c c c-2-\mathrm{Ox}_{2}$ in acetone or TFA without constrained protonation calculated using TD-DFT ${ }^{78,103,104}$ are shown in Figure 5 a (for details, see Computational Methods in Supplementary Information). The UV-vis spectra calculated for rccc-2$\mathrm{Ox}_{2}$ in TFA with initial constrained protonation of an acetyloxy $\mathrm{C}=\mathrm{O}$ group (Figure $5 \mathrm{~b}$, red line) followed by removal of the proton without structure relaxation (Figure $5 b$, blue line) show very good agreement with the experimentally obtained data (compare to Figures $3 b$ ). The small difference between the spectra in Figure $5 b$ indicates that any charge located at the acetyloxy group present due to protonation makes only a minor contribution to the resulting low-energy absorbance band in UV-vis. This effect is also supported by the plots of differential excitation densities (DED) prior to and after removal of the proton from the protonated form of $\mathrm{rccc}^{-2-O x_{2}}$, (Figures $5 \mathrm{c}-\mathrm{e}$ ). The low energy absorption bands in the 450 to $580 \mathrm{~nm}$ region originate from the hemiquinone moieties of rccc-2-Ox 2 . The effects of solvent environment were also tested using TD-DFT resulting in consistent significant absorptive transitions in the 410 to $580 \mathrm{~nm}$ region (Figure S18). A further assessment of the effects of acetyloxy protonation on the structure was carried out by MD simulations. The results indicate that protonation at an acetyloxy $\mathrm{C}=\mathrm{O}$ group causes certain structural variation of the $r c c c-$ 2-Ox 2 macrocycle so that the phenol-hemiquinone are almost coplanar (Figure S19a) and the central ring is increasingly skewed (Figure S19b). These structural changes are responsible for the emergence of the new low-energy absorption bands due to the resulting increase in $\pi$-electronic conjugation between hemiquinone and macrocycle. It should be noted that protonation on an acetyloxy group would be the first step in their acid promoted hydrolytic cleavage, although we found no evidence for decomposition of $\operatorname{rccc}-\mathbf{2}-\mathrm{Ox}_{2}$ by this route under the conditions used 
(treatment with strong mineral acids does result in decomposition to intractable products). Therefore, while protonation of acetyloxy groups in this system can influence the molecular conformation so that new low energy bands appear in UV-vis spectra, these protonated states are not sufficiently persistent to lead to deacetylation of the macrocycle.

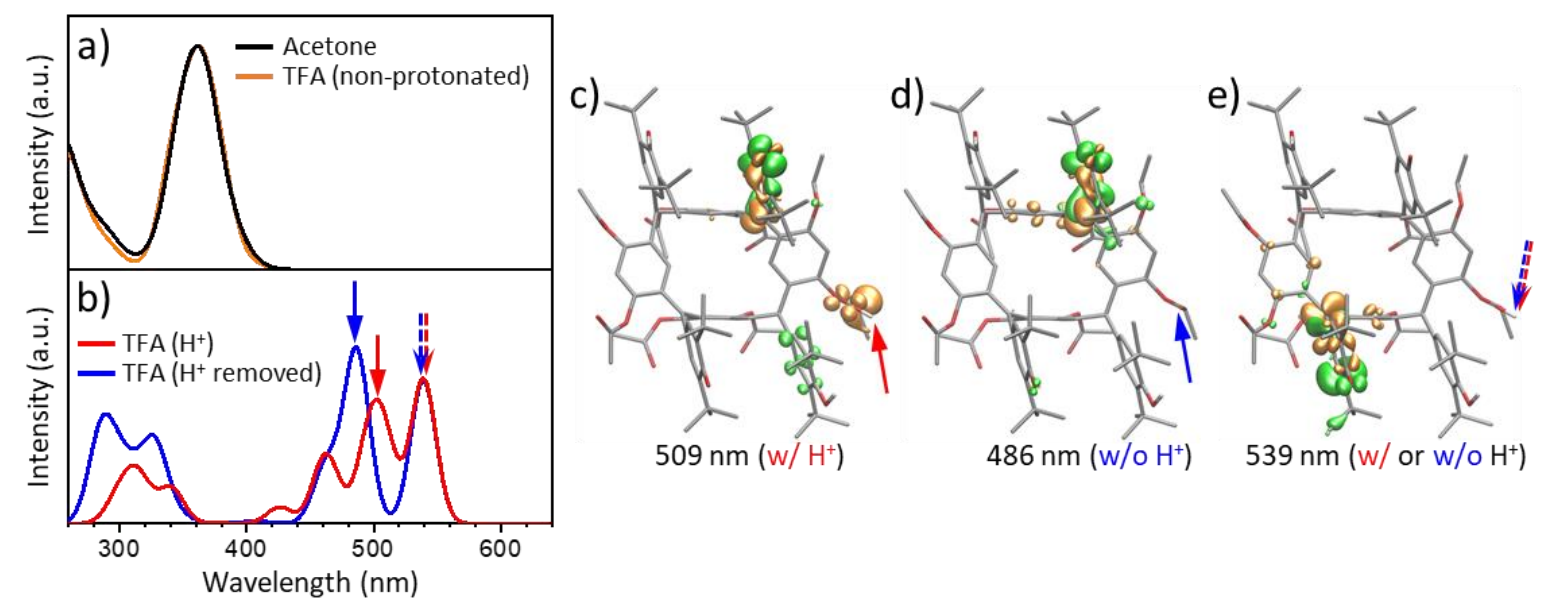

Figure 5. Calculated UV-vis spectra and related molecular conformations. TD-DFT (wB97XD 6-311G(d)) UV-vis spectra of rccc-2-Ox 2 calculated from 20 averaged statistically-independent snapshots from MD simulations performed under various conditions: (a) Non-protonated $r c c c-2-\mathrm{Ox}_{2}$ in neat acetone and TFA solvents. (b) Spectrum of $r c c c-2-\mathrm{Ox}_{2}$ in TFA with protonation constrained at one acetyloxy group and the spectrum after removal of the proton (without structure relaxation). MD snapshots of differential excitation density (DED) of various states of $r c c c-2-O x_{2}$ in TFA. Green isodensities show the negative regions from which electrons are excited while the positive orange isodensities indicate the regions to which the electrons are excited. Transitions shown are responsible for UV-vis absorptions at wavelengths indicated below each structure. (c) DED of acetyloxy protonated (location denoted by red arrow) rccc-2-Ox 2 leading to absorption at 509 $\mathrm{nm}$. (d) DED of rccc-2-Ox 2 after removal of proton from acetyloxy group in (c) (location denoted by blue arrow) shifts the absorption from $509 \mathrm{~nm}$ to $486 \mathrm{~nm}$. (e) DED of $r c c c-2-O x_{2}$ absorbance band at $539 \mathrm{~nm}$ indicates no change with or without protonation of the acetyloxy group (dashed red/blue arrows). The actual structure represents a protonated form. 
The calculations suggest that in TFA there is a degree of protonation or at least strong $\mathrm{H}$ bonding of acetyloxy $\mathrm{C}=\mathrm{O}$ groups of $r c c c-2-\mathrm{Ox}_{2}$, which influences its electronic structure, although the corresponding experimental observations suggest only incomplete or equilibrium processes. Also, the addition of stronger (than TFA) organic acids causes a further increase in the intensity of the absorption centred at $520 \mathrm{~nm}$ in experimentally observed spectra. Other pertinent experimental observations include infrared spectra (see Supplementary Information, Figure S20), where absorptions due to acetyloxy carbonyl stretching are shifted to lower frequency when exposed to TFA vapour, which can be associated with hydrogen bonding. There is also a weak ESR signal in rccc2-Ox $($ see Supplementary Information, Figure S16) although compounds containing multiple DtBHP groups usually contain small amounts of phenoxyl radical due to handling under aerobic conditions. The spectroscopic changes, including shifts in the ${ }^{13} \mathrm{C}$ resonances of the acetyloxy carbonyl groups and the appearance of the low energy UV-vis absorption, suggest that there is a significant protic process occurring between TFA and the acetyloxy $\mathrm{C}=\mathrm{O}$ groups. The strength of this interaction is sufficient to change the conformation of $r c c c-2-\mathrm{Ox}_{2}$, which cannot be detected directly. It also occurs in the proximity of a fuchsone moiety which is usually susceptible to protonation and rearrangement. In $r c c c-2-\mathrm{Ox}_{2}$, it seems then that two effects operate: (1) macrocyclic acetyloxy substituents are sufficiently electron-withdrawing to destabilize prospective meso-carbocations thus disfavouring protonation at the hemiquinonoid $\mathrm{C}=\mathrm{O}$, and (2) multiple acetyloxy groups of $r c c c-2-0 x_{2}$ are available for interaction with the acid medium, which leads to modification of its chromophore structure and emergence of electronic absorption bands in the visible region. It is currently unclear whether or not (2) influences (1) so that protonation at hemiquinonoid is even more strongly disfavoured at higher acidities. 
We note that the simple variation of macrocyclic substituent from benzyloxy (ether) to acetyloxy (ester) leads to a dramatic change in the response of the compounds to their environments. For the former (ether), meso-substituent protonation affects the properties of the compounds, while for the latter (ester) described here, interaction with the medium is limited to the macrocyclic acetyloxy (i.e., not meso) substituents. This is an important point as we learn about the factors affecting the chemistry of resorcinarenes and, in particular, the fuchsonarenes. As mentioned previously, the resorcinarenes have largely been considered for their propensity to form large nanocapsular assemblies. In this and previous work, we have found that complex chemical relationships exist, and these can occur not only intramolecularly between different types of substituents (e.g., macrocylic benzyloxy groups support meso-carbocation formation in contrast to acetyloxy) but also intermolecularly involving the solvent medium. It is interesting to note that in this case, the resistance to protonation of hemiquinonoid groups probably caused by macrocyclic acetyloxy groups is countered by a corresponding perhaps counterintuitive interaction of the acetyloxy groups with the acidic medium. Also, coincidentally, interactions of acid with fuchsonarenes at hemiquinonoid or acetyloxy groups lead to the emergence of UV-vis bands in the visible region despite the origins of these bands being very different.

\section{Conclusion}

In conclusion, we have demonstrated the activity of a fuchsonarene as a molecular single statordouble rotor. In terms of its structure, this is a rare example where rotary action can be controlled by varying solvent, temperature, or chemical inputs yielding a controllable system whose rotation rate can be tuned in the range of $2 \mathrm{~s}^{-1}$ to $20,000 \mathrm{~s}^{-1}$ depending upon the combination of the 
aforementioned inputs. Rotation speed governed by the TFA concentration can be monitored using UV-vis absorption data due to its correlation with the rotation rate determined by ${ }^{1} \mathrm{H} N M R$ spectroscopy, and suitable formulae for this purpose have been presented. TD-DFT was used to investigate features of this system and indicates that strong interactions of the acetyloxy groups with the solvent (most likely $\mathrm{H}$-bonding) occur in acidic solution and are responsible for the emergence of new low energy bands in the UV-vis spectrum of $r c c c-2-O x_{2}$. Overall, this work suggests a molecular mechanical method for estimating solution acidity by simple monitoring of UVvis spectra, and also gives insight into a possible method for modulating rotary activity in molecular machines.

\section{Data availability}

The experimental procedures, characterization data, and NMR spectra are provided in the Supplementary Information.

\section{Author contributions}

D. T. P., J. L. and J. P. H. designed the experiments, led the project and composed and wrote the manuscript with scientific discussions and contributions from all co-authors. D. T. P. performed the synthesis and chemical analyses of the compounds and collected analytical data. J. L. analyzed the $\mathrm{VT}^{1} \mathrm{H}$ NMR data, extracted the rate constants and constructed the calibration curves. Z. F. undertook the MD and DFT computational work. V. B. and L. H. performed SVD analysis. M. K. C. undertook UV-vis measurements and data processing. All authors contributed to the writing of the manuscript. 


\section{Conflicts of interest}

There are no conflicts to declare.

\section{Acknowledgements}

This work was partly supported by World Premier International Research Center Initiative (WPI Initiative), MEXT, Japan. D. T. P is grateful to the Japan Society for the Promotion of Science (JSPS) for a JSPS Fellowship and to the International Center for Young Scientists (ICYS-Namiki), NIMS for continued financial support. This work was also partially supported by JSPS KAKENHI Grant No. 19K05229. Computational resources were supplied by the project "e-Infrastruktura CZ" (e-INFRA LM2018140) provided within the program Projects of Large Research, Development and Innovations Infrastructures. 


\section{References}

1. V. Garcia-Lopez, D. Liu and J. M. Tour, Chem. Rev., 2020, 120, 79-124.

2. J. Michl and E. C. H. Sykes, ACS Nano, 2009, 3, 1042-1048.

3. G. S. Kottas, L. I. Clarke, D. Horinek and J. Michl. Chem. Rev., 2005, 105, 1285-1376.

4. G. Rapenne and C. Joachim, Top. Curr. Chem., 2014, 354, 253-277.

5. D. Lensen and J. A. A. W. Elemans, Soft Matter, 2012, 8, 9053-9063.

6. C. Ni and J.-Z. Wang, Surf. Rev. Lett., 2018, 25(Supp01), 1841004.

7. F. Cherioux, O. Galangau, F. Palmino and G. Rapenne, ChemPhysChem, 2016, 17, 1742-1751.

8. S. Erbas-Cakmak, D. A. Leigh, C. T. McTernan and A. L. Nussbaumer, Chem. Rev., 2015, 115, 1008110206.

9. S. Kassem, T. van Leeuwen, A. S. Lubbe, M. R. Wilson, B. L. Feringa and D. A. Leigh, Chem. Soc. Rev., 2017, 46, 2592-2621.

10. F. Lancia, A. Ryabchun and N. Katsonis, Nat. Rev. Chem., 2019, 3, 536-551.

11. R. D. Astumian, Acc. Chem. Res., 2018, 51, 2653-2661.

12. V. Balzani, A. Credi and M. Venturi, Molecular Devices and Machines: Concepts and Perspectives for the Nanoworld, Wiley VCH Weinheim, Germany, (2008).

13. V. Balzani, A. Credi, F. M. Raymo and J. F. Stoddart, Angew. Chem. Int. Ed. Engl., 2000, 39, 33483391.

14. E. R. Kay, D. A. Leigh and F. Zerbetto, Angew. Chem. Int Ed., 2007, 46, 72-191. 
16. M. Schmittel, Isr. J. Chem., 2019, 59, 197-208.

17. R. Herges, Chem. Sci., 2020, 11, 9048-9055.

18. A. B. Kolomeisky J. Phys.: Condens. Matter, 2013, 25, 463101.

19. M. Baba, K. Iwamoto, R. lino, H. Ueno, M. Hara, A. Nakanishi, J.-I. Kishikawa, H. Noji and K. Yokoyama, Proc. Nat. Acad. Sci. USA, 2016, 113, 11214-11219.

20. M. Baroncini, S. Silvi and A. Credi, Chem. Rev., 2020, 120, 200-268.

21. C. Cheng and J. F. Stoddart, ChemPhysChem, 2016, 17, 1780-1793.

22. M. Baroncini, L. Casimiro, C. de Vet, J. Groppi, S. Silvi and A. Credi, ChemistryOpen, 2018, 7, 169179.

23. H. A. Kistemaker, P. Stacko, J. Visser and B. L. Feringa, Nat. Chem., 2015, 7, 890-896.

24. A. Gerwien, P. Mayer and H. Dube, Nat. Commun., 2019, 10, 4449.

25. J. Echeverria, S. Monturet and C. Joachim, Nanoscale, 2014, 6, 2793-2799.

26. K. Sun, J.-L. Luo, X. Zhang, Z.-J. Wu, Y. Wang, H.-K. Yuan, Z.-H. Xiong, S.-C. Li, Q.-K. Xue and J.-Z. Wang, ACS Nano, 2017, 11, 10236-10242.

27. M. E. Howe and M. A. Garcia-Garibay, J. Org. Chem., 2019, 84, 9835-9849.

28. A. Comotti, S. Bracco and P. Sozzani, Acc. Chem. Res., 2016, 49, 1701-1710.

29. P. Martinez-Bulit, A. J. Stirk and S. J. Loeb, Trends Chem., 2019, 1, 588-600. 
30. C. H. Wolstenholme, H. Hu, S. Ye, B. E. Funk, D. Jain, C.-H. Hsiung, G. Ning, Y. Liu, X. Li and X. Zhang, J. Am. Chem. Soc., 2020, 142, 17515-17523.

31. W. L. Goh, M. Y. Lee, T. L. Joseph, S. T. Quah, C. J. Brown, C. Verma, S. Brenner, F. J. Ghadessy and Y. N. Teo. J. Am. Chem. Soc., 2014, 136, 6159-6162.

32. M. A. Haidekker and E. A. Theodorakis, J. Biol. Eng., 2010, 4, 11.

33. A. S. Kashirina, I. Lopez-Duarte, M. Kubankova, A. A. Gulin, V. V. Dudenkova, S. A. Rodimova, H. G. Torgomyan, E. V. Zagaynova, A. V. Meleshina and M. K. Kuimova, Sci. Rep., 2020, 10, 14063.

34. X. Liu, W. Chi, Q. Qiao, S. V. Kokate, E. P. Cabrera, Z. Xu, X. Liu and Y.-T. Chang, ACS Sensors, 2020, 5, 731-739.

35. T. Mori, H. Chin, K. Kawashima, H. T. Ngo, N.-J. Cho, W. Nakanishi, J. P. Hill and Katsuhiko Ariga, ACS Nano, 2019, 13, 2410-2419.

36. T. Mori, H. Komatsu, N. Sakamoto, K. Suzuki, J. P. Hill, M. Matsumoto, H. Sakai, K. Ariga and W. Nakanishi. Phys. Chem. Chem. Phys., 2018, 20, 3073-3078.

37. L. Feng, Y. Xie, S. K. Au-Yeung, H. B. Hailu, Z. Liu, Q. Chen, J. Zhang, Q. Pang, X. Yao, M. Yang, L. Zhang and H. Sun, Chem. Commun., 2020, 56, 8480-8483.

38. A. M. Desai and P. K. Singh, Chem. Eur. J., 2019, 25, 2035-2042.

39. Y. Wu, G. Wang, Q. Li, J. Xiang, H. Jiang and Y. Wang. Nat. Commun., 2018, 9, 1953.

40. P. K. Biswas, S. Saha, S. Gaikwad and M. Schmittel, J. Am. Chem. Soc., 2020, 142, 7889-7897.

41. P. K. Biswas, S. Saha, Y. Nanaji, A. Rana and M. Schmittel, Inorg. Chem., 2017, 56, 6662-6670. 
42. T. Ikeda, T. Tsukahara, R. lino, M. Takeuchi and H. Noji, Angew. Chem. Int. Ed., 2014, 53, 1008210085.

43. Z. Asfari, C. Naumann, G. Kaufmann and J. Vicens, Tetrahedron Lett., 1998, 39, 9007-9010.

44. J. Sturala, M. K. Etherington, A. N. Bismillah, H. E. Higginbotham, W. Trewby, J. A. Aguilar, E. H. C. Bromley, A.-J. Avestro, A. P. Monkman and P. R. McGonigal, J. Am. Chem. Soc., 2017, 139, 1788217889.

45. K. K. Kartha, A. Takai, Z. Futera, J. Labuta and M. Takeuchi, Angew. Chem. Int. Ed., 2021, 60, $16466-16471$.

46. C. M. A. Gangemi, A. Pappalardo and G. T. Sfrazzetto, Curr. Org. Chem., 2015, 19, 2281-2308.

47. P. Jin, S. J. Dalgarno and J. L. Atwood, Coord. Chem. Rev., 2010, 254, 1760-1768.

48. A. M. A. van Wageningen, W. Verboom and D. N. Reinhoudt, Pure Appl. Chem., 1996, 68, 12731277.

49. P. Timmerman, W. Verboom and D. N. Reinhoudt, Tetrahedron, 1996, 52, 2663-2704.

50. H. Erdtman, S. Högberg, S. Abrahamsson and B. Nilsson, Tetrahedron Lett., 1968, 9, 1679-1682.

51. H. Kitagawa, Y. Kobori, M. Yamanaka, K. Yoza and K. Kobayashi, Proc. Nat. Acad. Sci. U. S. A., 2009, 106, 10444-10448.

52. S. Harthong, B. Dubessy, J. Vachon, C. Aronica, J.-C. Mulatier and J.-P. Dutasta, J. Am. Chem. Soc., 2010, 132, 15637-15643.

53. A. G. S. Högberg, J. Am. Chem. Soc., 1980, 102, 6046-6050. 
54. R. H. Patil, C. Zhang and J. L. Atwood, Chem. Eur. J., 2016, 22, 15202-15207.

55. L. R. MacGillivray and J. L. Atwood, Nature, 1997, 389, 469-472.

56. J. L. Atwood, L. J. Barbour and A. Jerga, Proc. Nat. Acad. Sci. U. S. A, 2002., 99, 4837-4841.

57. Q. Zhang, L. Catti and K. Tiefenbacher, Acc. Chem. Res., 2018, 51, 2107-2114.

58. A. Pappalardo, R. Puglisi and G. T. Sfrazzetto, Catalysts, 2019, 9, 630.

59. B. W. Purse and J. Rebek Jr., Proc. Nat. Acad. Sci. U. S. A., 2005, 102, 10777-10782.

60. S. Pascal, L. Lavaud, C. Azarias, A. Varlot, G. Canard, M. Giorgi, D. Jacquemin and O. Siri. J. Org. Chem., 2019, 84, 1387-1397.

61. W. Maes and W. Dehaen, Chem. Soc. Rev., 2008, 37, 2393-2402.

62. H.-D. Becker, J. Org. Chem., 1967, 32, 2943-2947.

63. D. T. Payne, W. A. Webre, Y. Matsushita, N. Zhu, Z. Futera, J. Labuta, W. Jevasuwan, N. Fukata, J. S. Fossey, F. D’Souza, K. Ariga, W. Schmitt and J. P. Hill, Nat. Commun., 2019, 10, 1007

64. D. T. Payne, W. A. Webre, H. Gobese, Y. Matsushita, P. A. Karr, M. K. Chahal, J. Labuta, J. S. Fossey, K. Ariga, F. D'Souza and J. P. Hill, Chem. Sci., 2020, 11, 2614-2620.

65. L. R. Milgrom, Tetrahedron, 1983, 39, 3895-3898.

66. A. J. Golder, L. R. Milgrom, K. B. Nolan and D. C. Povey, J. Chem. Soc., Chem. Commun., 1989, 17511753.

67. L. R. Milgrom and J. P. Hill, J. Heterocycl. Chem., 1993, 30, 1629-1633. 
68. J. P. Hill, I. J. Hewitt, C. E. Anson, A. K. Powell, A. L. McCarthy, P. Karr, M. Zandler and F. D'Souza J. Org. Chem., 2004, 69, 5861-5869.

69. F. D’Souza, N. K. Subbaiyan, Y. Xie, J. P. Hill, K. Ariga, K. Ohkubo and S. Fukuzumi, J. Am. Chem. Soc., 2009, 131, 16138-16146.

70. S. Ishihara, J. P. Hill, A. Shundo, K. Ohkubo, S. Fukuzumi, M. R. J. Elsegood, S. J. Teat and K. Ariga, J. Am. Chem. Soc., 2011, 133, 16119-16126.

71. J. Labuta, Z. Futera, S. Ishihara, H. Kourilova, Y. Tateyama, K. Ariga and J. P. Hill, J. Am. Chem. Soc., 2014, 136, 2112-2118.

72. M. K. Chahal, D. T. Payne, J. Labuta, P. A. Karr, F. D’Souza, K. Ariga and J. P. Hill, Chem. Eur. J., 2020, 26, 13177-13183.

73. M. K. Chahal, A. Liyanage, A. Z. Alsaleh, P. A. Karr, J. P. Hill, and F. D’Souza, Chem. Sci., 2021, 12, 4925-4930.

74. H. S. Gutowsky and A. Saika, J. Chem. Phys., 1953, 21, 1688-1694.

75. V. Římal, H. Štěpánková and J. Štěpánek, Concepts Magn. Reson. Part A, 2011, 38, 117-127.

76. S. Glasstone, K. J. Laidler and H. Eyring, The Theory of Rate Processes, pp. 199 (McGraw-Hill Book Company, New York and London, 1941).

77. J. Wang, R. M. Wolf, P. A. Kollman and D. A. Case, J. Comput. Chem., 2004, 25, 1157-1174.

78. Gaussian 16, Revision C.01, M. J. Frisch, G. W. Trucks, H. B. Schlegel, G. E. Scuseria, M. A. Robb, J. R. Cheeseman, G. Scalmani, V. Barone, G. A. Petersson, H. Nakatsuji, X. Li, M. Caricato, A. V. Marenich, J. Bloino, B. G. Janesko, R. Gomperts, B. Mennucci, H. P. Hratchian, J. V. Ortiz, A. F. 
Izmaylov, J. L. Sonnenberg, D. Williams-Young, F. Ding, F. Lipparini, F. Egidi, J. Goings, B. Peng, A. Petrone, T. Henderson, D. Ranasinghe, V. G. Zakrzewski, J. Gao, N. Rega, G. Zheng, W. Liang, M. Hada, M. Ehara, K. Toyota, R. Fukuda, J. Hasegawa, M. Ishida, T. Nakajima, Y. Honda, O. Kitao, H. Nakai, T. Vreven, K. Throssell, J. A. Montgomery, Jr., J. E. Peralta, F. Ogliaro, M. J. Bearpark, J. J. Heyd, E. N. Brothers, K. N. Kudin, V. N. Staroverov, T. A. Keith, R. Kobayashi, J. Normand, K. Raghavachari, A. P. Rendell, J. C. Burant, S. S. Iyengar, J. Tomasi, M. Cossi, J. M. Millam, M. Klene, C. Adamo, R. Cammi, J. W. Ochterski, R. L. Martin, K. Morokuma, O. Farkas, J. B. Foresman and D. J. Fox, Gaussian, Inc., Wallingford CT, 2016.

79. H. J. C. Berendsen, D. van der Spoel and R. van Drunen, Comp. Phys. Comm., 1995, 91, 43-56.

80. GROMACS 2020 Manual, E. Lindahl, M. J. Abraham, B. Hess and D. van der Spoel, DOI: 10.5281/zenodo.3562512.

81. S. Nose, J. Chem. Phys., 1984, 81, 511-519.

82. W. G. Hoover, Phys. Rev. A, 1985, 31, 1695-1697.

83. T. Darden, D. York and L. Pedersen, J. Chem. Phys., 1993, 98, 10089-10092.

84. U. Essmann, L. Perera and M. L. Berkowitz, J. Chem. Phys., 1995, 103, 8577-8593.

85. G. M. Torrie and J. P. Valleau, J. Comput. Phys., 1977, 23, 187-199.

86. S. Kumar, D. Bouzida, R. H. Swendsen, P. A. Kollman and J. M. Rosenberg, J. Comput. Chem., 1992, 13, 1011-1021.

87. A. Mazzanti, L. Lunazzi, M. Minzoni and J. E. Anderson, J. Org. Chem., 2006, 71, 5474-5481. 
88. C. J. Medforth, R. E. Haddad, C. M. Muzzi, N. R. Dooley, L. Jaquinod, D. C. Shyr, D. J. Nurco, M. M. Olmstead, K. M. Smith, J.-G. Ma and J. A. Shelnutt, Inorg. Chem., 2003, 42, 2227-2241.

89. E. R. Henry and J. Hofrichter, ed. B.-M. in Enzymology, Academic Press, 1992, vol. 210, pp. 129192.

90. E. R. Malinowski, Factor Analysis in Chemistry, Wiley, 2002.

91. P. Mojzes, P. Praus, V. Baumruk, P.-Y. Turpin, P. Matousek and M. Towrie, Biopolymers 2002, 67, 278-281.

92. J. Palacký, P. Mojzeš and J. Bok, J. Raman Spectrosc., 2011, 42, 1528-1539.

93. V. Březina, S. Ishihara, J. Lang, L. Hanyková, K. Ariga, J. P. Hill and J. Labuta, ChemistryOpen, 2018, 7, 323-335.

94. D. T. Payne, M. K. Chahal, V. Březina, W. A. Webre, K. Ariga, F. D'Souza, J. Labuta and J. P. Hill, Front. Chem. Sci. Eng., 2020, 14, 28-40.

95. M. K. Chahal, J. Labuta, V. Březina, P. A. Karr, Y. Matsushita, W. A. Webre, D. T. Payne, K. Ariga, F. D'Souza and J. P. Hill, Dalton Trans., 2019, 48, 15583-15596.

96. K. Naoda, D. Shimizu, J. O. Kim, K. Furukawa, D. Kim and A. Osuka, Chem. Eur. J., 2017, 23, 89698979.

97. D. Shimizu and A. Osuka, Chem. Sci., 2018, 9, 1408-1423.

98. C. Fraser and R. D. Young, J. Org. Chem., 2018, 83, 505-509. 
99. B. Kim, G. Storch, G. Banerjee, B. Q. Mercado, J. Castillo-Lora, G. W. Brudwig, J. M. Mayer and S. J. Miller, J. Am. Chem. Soc., 2017, 139, 15239-15244.

100. O. B. Youchret-Zallez, S. Besbes-Hentati, M. Bouvet and H. Said, J. Inclusion Phenom. Macrocyclic Chem., 2014, 79, 383-390.

101. S. Meddeb-Limem, S. Besbes-Hentati, H. Said and M. Bouvet, Electrochim. Acta, 2011, 58, 372382.

102. P. R. Bangal, Chem. Phys. Lett., 2005, 401, 200-204.

103. M. E. Casida, C. Jamorski, K. C. Casida and D. R. Salahub, J. Chem. Phys., 1998, 108, 4439-4449.

104. J.-D. Chai and M. Head-Gordon, Phys. Chem. Chem. Phys., 2008, 10, 6615-6620. 